\section{G577(P) TARGETED NEONATAL ECHOCARDIOGRAPHY}

${ }^{1}$ A Burman, ${ }^{2} \mathrm{~S}$ Khan, ${ }^{3} \mathrm{~A}$ Khushu, ${ }^{2} \mathrm{~W}$ Kelsall. ${ }^{1}$ Acute Medical Unit, West Suffolk Hospital NHS Trust, Bury St Edmunds, UK; ${ }^{2}$ Paediatrics, Addenbrooke's, Cambridge, UK; ${ }^{3}$ Paediatrics, Watford General Hospital, Watford, UK

\subsection{6/archdischild-2020-rcpch.494}

Introduction The incidence of congenital heart disease (CHD) varies between 6/1000 live births for moderate/severe CHD to $75 / 1000$ for trivial lesions e.g. small muscular ventricular septal defects. ${ }^{1}$ There are no guidelines for which neonates undergoing non-cardiac surgery require a pre-operative echocardiogram (POE). Paediatric anaesthetists and surgeons often request for stable babies to have a POE before surgery. In this unit, paediatricians with expertise in cardiology (PECs) perform these scans, often being approached out of hours.

Aims To review the cardiovascular status of babies undergoing surgery before 28 days corrected age and determine if the clinical examinations correlated with POE.

Methods Patients undergoing non-cardiac surgery less than 1 month corrected age between April 2015 - 2018 were identified using the Trust Theatreman system. Data was subsequently collected from the Badgernet and Epic systems.

Results We reviewed 270 surgical events in 235 patients. 173 (74\%) had a normal clinical examination and 125 (53\%) had POE. Of the 173 babies with normal examinations 71 (41\%) had a POE, which was abnormal in only $6(3 \%)$. In the 62 babies with an abnormal examination, a POE was performed in $54(87 \%)$ babies with $14(26 \%)$ having more significant CHD. Of the 125 POE only $16 \%$ were abnormal.

Conclusions There was a good correlation between normal examination and normal POE. An abnormal examination was more frequently associated with an abnormal POE. In developing local guidelines, we would hope to agree that a POE is not required in patients with normal examinations. Such patients could safely have echocardiography screening postoperatively.

\section{G578(P) PROGNOSTIC ACCURACY OF MAGNETIC RESONANCE SPECTROSCOPY (MRS) BIOMARKERS IN NEONATES WITH HYPOXIC ISCHAEMIC ENCEPHALOPATHY (HIE): A SYSTEMATIC REVIEW AND META-ANALYSIS OF DIAGNOSTICS}

${ }^{1} \mathrm{CR}$ Rizava, ${ }^{2} \mathrm{GN}$ Nikolaidis, ${ }^{3} \mathrm{DC}$ Connolly, ${ }^{4} \mathrm{AH}$ Hart. ${ }^{1}$ Department of Neonatology, Sheffield Teaching Hospitals NHS FT, Sheffield, UK; ${ }^{2}$ Center of Health Economics, University of York, York, UK; ${ }^{3}$ Department of Paediatric Neuroradiology, Sheffield Children's Hospital NHS FT, Sheffield, UK; ${ }^{4}$ Department of Paediatric Neurology, Sheffield Children's Hospital NHS FT, Sheffield, UK

\subsection{6/archdischild-2020-rcpch.495}

Introduction Magnetic Resonance Spectroscopy (MRS) biomarkers have been used for prognostication of neurodevelopmental outcomes in newborns affected by Hypoxic Ischaemic Encephalopathy (HIE). Previous studies have evaluated these markers primarily in non-cooled infants with HIE. ${ }^{1}$ Here, we aim to also include recent studies on cooled infants and metaanalyse the full evidence base, to obtain updated predictive accuracy estimates of MRS biomarkers and explore the impact of cooling therapy on heterogeneity.

Methods We systematically searched MEDLINE, COCHRANE, EMBASE and CINHAL for studies assessing MRS biomarkers for prognostication of adverse neurodevelopmental outcome (assessed $\geq 12$ months of age) or death in infants ( $\geq 36$ weeks gestation) affected by HIE. Data were extracted from eligible studies and meta-analysed using univariate approaches in RStudio with the madad package. We assessed the impact of effect modulators such as therapeutic hypothermia and strength of the magnetic field by means of subgroup analysis. We rated the methodological quality of the included studies using the QUADAS-2 tool.

Results We retrieved 72 eligible studies of which 25 reported outcomes on prognostic accuracy of MRS biomarkers; five of which were performed in infants who had received TH. The biomarker lactate (lac) to N-acetylacetate (NAA) -lac/NAAratio, measured in the basal ganglia, had a pooled sensitivity of 0.91 (95\% CI : $0.82-0.95)$ and a pooled specificity of 0.90 (95\% CI:0.81 - 0.95) in predicting neurodevelopmental outcomes. The rest of the biomarkers, namely lac/cr (creatinine), NAA/cr and NAA/cho (choline) had similar pooled estimates, with mean sensitivity ranging from 0.66 to 0.77 and specificity from 0.8 to 0.91 . Subgroup analysis revealed increased mean sensitivity of lac/NAA in infants who received hypothermia compared to those who did not $(0.96$ and 0.86 respectively, $\mathrm{p}$-value $=0.32$ ). The specificity of the test remained similar $(0.88$ and 0.91 respectively, $p$-value $=0.61)$.

Conclusion Lac/NAA measured by MRS can accurately predict adverse neurodevelopmental outcomes in infants with HIE. Therefore, Lac/NAA may be a useful surrogate marker for studies evaluating new therapies for HIE.

\section{REFERENCES}

1. Thayyil S, Chandrasekaran M, Taylor A, Bainbridge A, Cady EB, Chong WK, et al. Cerebral magnetic resonance biomarkers in neonatal encephalopathy: a metaanalysis. Pediatrics. 2010;125(2):e382-95

\section{G579(P) CAN THE ONLINE WINROP ALGORITHM PREDICT THE DEVELOMENT OF SEVERE RETINOPATHY OF PREMATURITY IN A MULTI-ETHNIC POPULATION OF PRETERM BABIES?}

R Bhudia, SM Husain. Centre for Genomics and Child Health, Queen Mary University of London, London, UK

\subsection{6/archdischild-2020-rcpch.496}

Retinopathy of prematurity (ROP), a retinal condition of the preterm retina, can cause blindness if undetected and untreated. Babies at risk ( $<32 \mathrm{w}$ GA and/or $<1501 \mathrm{~g}$ BW) undergo screening to detect ROP requiring treatment (ROP-T). Over $95 \%$ of at risk babies do not develop ROP-T ${ }^{1}$ but are screened and utilise skilled resources. The online algorithm WINROP uses GA, BW and serial weights to identify early babies who subsequently develop ROP-T. It has the potential to improve ROP screening service delivery but it was developed and has been validated in mainly White populations. We investigated WINROP's ability to identify babies from a multiethnic population who subsequently developed ROP-T.

This service delivery improvement project was performed in a single NICU using retrospective data. Babies at risk of ROP, born between 01/09/2015 and 30/06/2016, and admitted to the NICU at $<48$ hours of age were identified. Data on GA, BW, and serial weights within the first 6 weeks of life were entered into WINROP. Its sensitivity and specificity for identifying babies who subsequently developed ROP-T was calculated. 
107 seven infants were included. Self-reported maternal ethnicity of the cohort was 37\% Black, 29\% White, 15\% Asian, 15\% Other, 3\% Mixed, 1\% Unknown. WINROP's sensitivity and specificity for identifying babies who subsequently developed ROP-T were $100 \%$ and $54 \%$ respectively. The positive predictive and negative predictive values were $17 \%$ and $100 \%$, respectively.

In this small, multi-ethnic cohort, WINROP identified all babies who subsequently developed ROP-T. WINROP might have a role in improving ROP screening service delivery but further work is required to confidently validate its performance.

\section{REFERENCES}

1. Adams GGW, Bunce C, Xing W, et al. Treatment trends for retinopathy of prematurity in the UK: active surveillance study of infants at risk. BMJ Open 2017;7: e013366.doi:10.1136/bmjopen-2016-013366.

\section{British paediatric allergy immunity and infection group (BPAllG)}

\section{G580 A 5-YEAR AUDIT OF THE DIAGNOSIS OF MALARIA IN PAEDIATRIC ACCIDENT AND EMERGENCY}

N Patel, C Douch, S Wazir. Paediatrics, Homerton University Hospital NHS FT, London, UK

10.1136/archdischild-2020-rcpch.497

Aims With vague presenting features, malaria can be difficult for clinicians to diagnose. The current guideline recommends 3 -day screening to rule out malaria. We aimed to audit the presentation and investigation of children with suspected malaria in Accident and Emergency between February 2014 January 2019, with comparison of the accuracy of one versus three screens.

Methods Retrospective data was collected from the electronic patient record of all children investigated for malaria between 2014-2019. Data collected included demographics, travel destination, laboratory results and number/results of malaria screens. The reference standard to diagnose malaria was Malaria Reference Laboratory confirmation. Malaria risk was determined using www.fitfortravel.nhs.uk. Fisher's exact test was used for association between categorical variables, with $P<0.05$ considered statistically significant.

Results 205 children were investigated for malaria from 20142019 , with $63 \%$ travelling to a high-risk destination. Only $14 \%$ had three consecutive screens; $64 \%$ had one, $16 \%$ had two and $6 \%$ had $>3$ non-consecutive screens.

19 children had malaria (90\% falciparum), and 95\% had travelled to Africa. Travellers to a high-risk destination were much more likely to contract malaria (odds ratio 9.90, $P<0.05)$. Fever was the universal symptom $(100 \%)$, with vomiting (47\%), headache (37\%) and anorexia (37\%) common presentations.

All initial screens included a rapid diagnostic test (RDT) and blood smear, with every case detected on day one. $P$. falciparum RDT had the best accuracy, with $100 \%$ sensitivity and negative predictive value $(\mathrm{NPV})(P<0.001)$. There was no significant difference between 1 screen $(n=132)$ and 3 consecutive screens $(n=28)$ for sensitivity, specificity, NPV and positive predictive value $(P<0.05)$. Nobody traveling to a low-risk destination contracted malaria, and the NPV of both microscopy and $P$. falciparum RDT was $100 \%$ in this group $(P<0.001)$.

Conclusion Malaria should always be considered in any febrile child that has traveled to a malaria-endemic region. We have modified our guidelines to recommended that a single screen is sufficient to exclude malaria in low-risk travellers where malaria is not strongly suspected. In those that have travelled to a high-risk destination or in whom malaria is strongly suspected, three consecutive screens should remain gold-standard.

\section{G581 IMPACT OF ROTAVIRUS VACCINATION ON INCIDENCE OF INTUSSUSCEPTION IN ENGLAND: A TEN-YEAR ANALYSIS}

${ }^{1} \mathrm{~L}$ McGeoch, ${ }^{1} \mathrm{~A}$ Finn, ${ }^{2} \mathrm{R}$ Marlow. ${ }^{1}$ Population Health Sciences, University of Bristol, Bristol, UK; ${ }^{2}$ Paediatric Emergency Department, Bristol Royal Children's Hospital, Bristol, UK

\subsection{6/archdischild-2020-rcpch.498}

Aims Rotavirus vaccination (RV) has been linked to a small increase in the risk of intussusception. It is unclear if $\mathrm{RV}$ is causative or precipitates events that would have happened anyway. We sought to determine whether introduction of RV was associated with a change in the overall and age group-specific incidence of childhood intussusception in England.

Methods Using hospital episode statistics for the entire English paediatric population, we used modified Poisson regression to compute intussusception incidence rate ratios (IRR) for the post-vaccine period (2014-2017) compared to the pre-vaccine period (2008-2012) in 0-12 months and 0-36 months age groups and in subgroups 8-16 weeks (point of vaccination), 17-24 weeks, 25-32 weeks, and 33-40 weeks. We also conducted interrupted time series (ITS) analysis to compute IRRs in the $0-12$ months and $0-36$ months age groups accounting for baseline trends.

Results Incidence rates and IRRs for intussusception in the post-vaccine period versus the pre-vaccine period are shown in table 1.

\begin{tabular}{llll} 
Abstract G581 Table 1 & \multicolumn{2}{l}{} \\
\hline Age Group & \multicolumn{2}{l}{ Incidence per 100,000 } & \multicolumn{2}{l}{ IRR CI) } \\
\cline { 2 - 4 } & 2008-2012 & $2014-2017$ & $0.86(0.78-0.94)$ \\
\hline 0-12 months & 33.0 & 28.3 & $1.46(1.12-1.91)$ \\
8-16 weeks & 17.9 & 26.3 & $0.77(0.63-0.94)$ \\
17-24 weeks & 44.6 & 34.4 & $0.71(0.59-0.86)$ \\
25-32 weeks & 51.2 & 36.5 & $0.94(0.76-1.15)$ \\
33-40 weeks & 38.9 & 36.5 & $0.86(0.80-0.93)$ \\
0-36 months & 16.0 & 13.8 & \\
\hline
\end{tabular}

Using ITS analysis, IRRs did not change significantly in the 0-12 months $(0.88,0.74-1.05)$ or $0-36$ months $(0.90,0.78-$ 1.03) age groups.

Conclusion Monovalent RV in England has not resulted in an overall increase in the incidence of intussusception. A transitory increase in the peri-vaccination age group is compensated for by a reduction in older infants, suggesting that RV may reveal individuals already predisposed to intussusception. 